\title{
Estudiantes Pragmáticos, Teóricos y Educación Superior Mediante Metodologías Activas
}

\author{
Pragmatists, Theorists' Students and Tertiary Education Through Active Methodologies
}

\author{
Erik Dreyer¹; Pilar Barahona² \& Bastián Chávez³
}

DREYER, E.; BARAHONA, P. \& CHÁVEZ, B. Estudiantes pragmáticos, teóricos y educación superior mediante metodologías activas. Int. J. Odontostomat., 15(1):152-158, 2021.

RESUMEN: Nuevos modelos de educación en salud, enfatizan la importancia del contexto y contenidos para la implementación de acciones significativas en terapéutica. Los estudiantes deben involucrarse activamente en su aprendizaje. Una manera de estimular este cambio son las metodologías activas de educación. Estudiantes educados mediante Aula Reversa (AR) y Aprendizaje Basado en Problemas (ABP) han demostrado destrezas profesionales superiores y aprendizaje efectivo. Según Honey-Alonso, hay cuatro estilos de aprendizaje: Teórico $(T)$, Pragmáticos $(P)$, Reflexivos (R) y Activos (A).Propósito:Implementar metodologías activas para profesionales, conociendo su estilo de aprendizaje, cuando cursan un programa de especialización. El programa de especialización en periodoncia de la Facultad de Odontología de la U. de Chile, posee la unidad de rehabilitación en periodonto reducido (URPR). Entre 2009-2019 tuvo 67 estudiantes, 31firmaron consentimiento informado. En URPR se implementó AR y ABP para: gestión del riesgo cariogénico, ferulización, desprogramación, ajuste oclusal, retención de dientes y selección de sitio para implantes. Se compararon la primera y última entrega de los ABP's categorizando contenidos disciplinares e interdisciplinares. En los mismos tiempos se aplicó el cuestionario Honey-Alonso (H-A). Se registraron los tiempos de la actividad. Datos analizados en SPSS v.21. Para la variable contenidos disciplinares e interdisciplinares se utilizó la prueba t de student, $\leq 0,05$. La cohorte $n=31,14$ mujeres y 17 hombres. $H-A$ inicial= $20(P)$ y $11(T) ; H-A$ final $=17(R), 8(A), 3(P)$ y $3(T)$. La media de contenidos disciplinares iniciales $(I)=8,10$ y finales $(F)=13,71 ; p=0,000$. La media de contenidos interdisciplinares $I=$ 2,48 y $F=13,19 ; p=0,000$. Diferencias de medias contenidos disciplinares $F-I=5,61$; interdisciplinares $F-I=10,71 p=0,000$. Todos los estudiantes fueron capaces de desarrollar metodologías activas, independiente de su estilo de aprendizaje y aumentaron significativamente más contenidos interdisciplinarios en contexto, para la toma de decisiones clínicas.

PALABRAS CLAVE: aprendizaje basado en problemas, educación dental, contenido, contexto.

\section{INTRODUCCIÓN}

Las competencias profesionales son esenciales para entregar respuesta a las necesidades terapéuticas en el área de la salud (Balogh et al., 2015; Christensen et al., 2017; Cutrer et al., 2017). Estas requieren que el dentista utilice una red integrada de conocimientos para identificar y resolver los variados problemas clínicos (Dalton et al., 2015; Forés et al., 2015; Gruppen, 2017). Los nuevos modelos conceptuales para la educación en salud enfatizan la importancia del contexto y contenidos para la implementación de ac- ciones significativas en terapéutica (Yazdani et al., 2017; Khin-Htun \& Kushairi, 2018).

Los programas de especialización en Odontología son esencialmente disciplinares y sus títulos son descriptores de ello. Es por ello, que los estudiantes utilizan fundamentalmente contenidos de la disciplina que están estudiando, al momento de tomar decisiones clínicas terapéuticas sin considerar la naturaleza contexto-dependiente de la toma de de-

\footnotetext{
${ }^{1}$ Cirujano-Dentista, PhD, Especialista en Rehabilitación Oral. Departamento de Odontología Conservadora, Facultad de Odontología, Universidad de Chile, Santiago, Chile.

${ }^{2}$ Cirujano-Dentista, MSc. Dirección de Pregrado, Facultad de Odontología, Universidad de Chile, Santiago, Chile.

${ }^{3}$ Sociólogo, Pontificia Universidad Católica de Chile, Santiago, Chile.
} 
cisiones clínicas en acción (Tovar-Moncada \& Crespo-Knopfler, 2015; Varpio et al., 2017; Martínez, 2018; Naranjo, 2018). Sin embargo, los problemas de salud oral son multidimensionales, simultáneos y no lineales, vale decir, las enfermedades y condiciones orales están presentes al mismo tiempo, en distintos grados de progresión y severidad (Glick et al., 2017).

La evidencia publicada enfatiza, como significativo para una aplicación posterior, la forma en que los contenidos son inicialmente desarrollados (Woods et al., 2007), la forma en son relacionados (de Bruin et al., 2005, Novak et al., 2006; Auclair, 2007), y la forma en que son almacenados, para ser recuperados en un uso posterior. Bajo esta perspectiva, los estudiantes de pre y postítulo deben dejar atrás el rol pasivo en su educación e involucrarse activamente en su aprendizaje.

Aula reversa (AR) (The Flipped Classroom, 2019) es un modelo pedagógico que transfiere el trabajo de determinados procesos de aprendizaje fuera del aula y utiliza el tiempo de clase, junto con la experiencia del docente, para facilitar y potenciar otros procesos de adquisición y práctica de conocimientos dentro del aula, para mejorar su comprensión conceptual. El aprendizaje basado en problemas (ABP) es una metodología educacional activa, en la cual el estudiante aprende mediante resolución de problemas facilitados. En ABP el estudiante centra su aprendizaje en un problema complejo que no tiene una única respuesta correcta. Los estudiantes pueden trabajar de manera colaborativa para identificar qué necesitan aprender para resolver el problema. Ellos se engranan en un aprendizaje autodirigido (AAD) y aplican su nuevo conocimiento al problema y refleja lo aprendido junto con la efectividad de la estrategia utilizada. El académico actúa como facilitador del proceso de aprendizaje en lugar de entregar el conocimiento. Los objetivos del ABP incluyen, ayudar al estudiante a desarrollar conocimiento flexible, destrezas efectivas para resolver problemas, destrezas de AAD, destrezas de colaboración efectiva y motivación intrínseca (Hmelo-Silver, 2004).

Existe evidencia publicada del uso de ABP en educación médica desde hace cerca de cuarenta años, sin embargo, su aplicación en odontología es reciente. Ella demuestra que los estudiantes educados mediante ABP demuestran destrezas profesionales superiores y aprendizaje efectivo cuando se comparan con otros, educados con metodologías tradicionales (Alrahlah, 2016). Su aplicación en la for- mación de especialistas en Odontología está pobremente representada. El presente ensayo tuvo como propósito observar el resultado de la implementación de AR y ABP en profesionales, a los que conocemos su denominado estilo de aprendizaje, cuando cursan un programa de especialización odontológico.

\section{MATERIAL Y MÉTODO}

El programa de especialización en periodoncia de la Facultad de Odontología de la U. de Chile, es el único programa de especialización en periodoncia del país, que posee una unidad de rehabilitación en periodonto reducido (URPR) con presencia en la malla curricular, durante los tres años del eje formativo. Los variados comités académicos y profesores responsables del programa durante los últimos diez años asignaron 50 horas presenciales para la URPR. Este programa fue recientemente acreditado por cuatro años por la Comisión Nacional de Acreditación de Chile (CNA Chile). La URPR ha tenido como objetivos de aprendizaje, activar los contenidos interdisciplinares entregados durante su formación de pregrado, actualizarlos, complejizarlos y ponerlos en contexto en los casos a resolver por los estudiantes. Durante el decenio 2009-2019 el programa tuvo un total de 67 estudiantes, profesionales Cirujano-Dentistas que desean continuar su formación para llegar a ser especialistas en periodoncia.

El docente a cargo de la URPR diseñó las sesiones teóricas para implementar AR y ABP. La arquitectura de las sesiones comprendió un breve encuadre, por parte del docente de los contenidos a tratar con entrega anticipada de la bibliografía de respaldo y pauta de lectura, con el propósito de generar discusión de grupo pequeño sobre los contenidos abordados en los artículos. Los énfasis en la bibliografía de respaldo se centraron en la línea de tiempo y el diseño metodológico de los artículos, todos relacionados con los contenidos a tratar. Los contenidos abordados fueron: gestión del riesgo cariogénico, ferulización, desprogramación, ajuste oclusal, retención de dientes y selección de sitio para implantes. Luego de un receso se continuaba con la presentación pauteada (seis imágenes) de un caso clínico por alumno, generando un clima en aula que estimuló la reflexión individual y colectiva. Finalmente se generó un proceso de puesta en contexto de los contenidos en el caso presentado mediante sistematización e integración, generando diversas pro- 
puestas terapéuticas. Esto se repitió en todas las sesiones asignadas para la URPR durante los tres años del eje formativo lo que permitió evaluar el progreso de los casos.

Para estudiar el desarrollo de los ABPs y contrastarlo con los denominados estilos de aprendizaje, se invitó a la totalidad de la cohorte de estudiantes del decenio a participar mediante la premisa: transformaremos sus casos clínicos en ABP y así aportaremos a la resolución clínica de ellos. Para identificar los denominados estilos de aprendizaje se utilizó el cuestionario Honey-Alonso (H-L). Solo 31 estudiantes aceptaron participar del ensayo, quienes firmaron consentimiento informado. Las observaciones se realizaron en condición inicial (basal) y cuando el alumno dio de alta al paciente (final). Las altas, y su derivación a terapia periodontal de soporte comenzaron a ocurrir a partir del término del segundo semestre del programa. Tanto en la primera y última entrega de los ABPs, se categorizaron los contenidos disciplinares e interdisciplinares que respaldaron las decisiones clínicas implementadas. Se comparó la cantidad de contenidos disciplinares e interdisciplinares entre la primera y ultima entrega de los ABPs. A su vez, en los mismos tiempos se aplicó el cuestionario Honey-Alonso. También se registraron los tiempos totales invertidos en la actividad. Los datos obtenidos fueron analizados mediante estadística descriptiva e inferencial, con el software SPSS v.21.El análisis aborda la variable cantidad de contenidos disciplinares e interdisciplinares al inicio y final de los ABPs, aplicándola prueba t de student con significancial $\leq a$ 0,05.

\section{RESULTADOS}

Las características de la cohorte en términos de edad y género se observan en la Tabla I. La cohorte está compuesta por 31 participantes: 14 mujeres (45\%) y 17 hombres (55\%). El total de la cohorte tuvo 30 años de edad en promedio, para las mujeres el promedio de edad fue de 30 años y en los hombres 29 años.

Tabla I. Características de la cohorte según género y edad.

\begin{tabular}{ccccc}
\hline & & Femenino & Masculino & Global \\
\hline \multirow{2}{*}{ Cohorte } & $\mathrm{N}$ & 14 & 17 & 31 \\
& $\%$ & $45 \%$ & $55 \%$ & $100 \%$ \\
\multirow{4}{*}{ Edad } & Media & 30 & 29 & 30 \\
& Rango & $27-36$ & $24-34$ & $24-36$ \\
\hline
\end{tabular}

154
Según Honey-Alonso, son cuatro estilos de aprendizaje: Teórico $(T)$, Pragmáticos $(P)$, Reflexivos $(R)$ y Activos (A). Los resultados de la aplicación inicial del cuestionario $\mathrm{H}-\mathrm{A}$ demostraron que, de los 31 estudiantes de la cohorte, 20 estudiantes fueron identificados como Pragmáticos y 11 como Teóricos Los resultados de la aplicación final del cuestionario demostraron que 8 estudiantes fueron identificados como Activos, 3 como Pragmáticos, 17 como Reflexivos y 3 como Teóricos (Tabla II).

Tabla II. Resultado de la aplicación del questionario HoneyAlonso en Inicial y Final

\begin{tabular}{ccccc}
\hline & \multicolumn{2}{c}{ H A In icial } & \multicolumn{2}{c}{ H A Final } \\
& $\mathrm{N}$ & $\%$ & $\mathrm{~N}$ & $\%$ \\
\hline $\mathrm{A}$ & - & - & 8 & $25,8 \%$ \\
$\mathrm{P}$ & 20 & $64,5 \%$ & 3 & $9,7 \%$ \\
$\mathrm{R}$ & - & - & 17 & $54,8 \%$ \\
$\mathrm{~T}$ & 11 & $35,5 \%$ & 3 & $9,7 \%$ \\
Total & 31 & $100 \%$ & 31 & $100 \%$ \\
\hline
\end{tabular}

La comparación de la aplicación del cuestionario Honey-Alonso, al inicio y al final de los ABPs expresados en frecuencia, se pueden observar en la Tabla III. Los estudiantes que comenzaron (HAI) como Pragmáticos, en su mayoría (85\%) finalizaron (HAF) en Reflexivo. Solo un $15 \%$ se mantuvo en Pragmático en ambas mediciones. Quienes comenzaron Teóricos, en un $72,7 \%$ cambiaron a Activos. Solo un 27,3 \% se mantuvo Teórico en ambas mediciones. Los cambios de la distribución de la cohorte en la gráfica de Honey-Alonso en condición inicial y final se pueden observar en La Figura 1.

\section{Estilos de aprendizaje inicial y final}

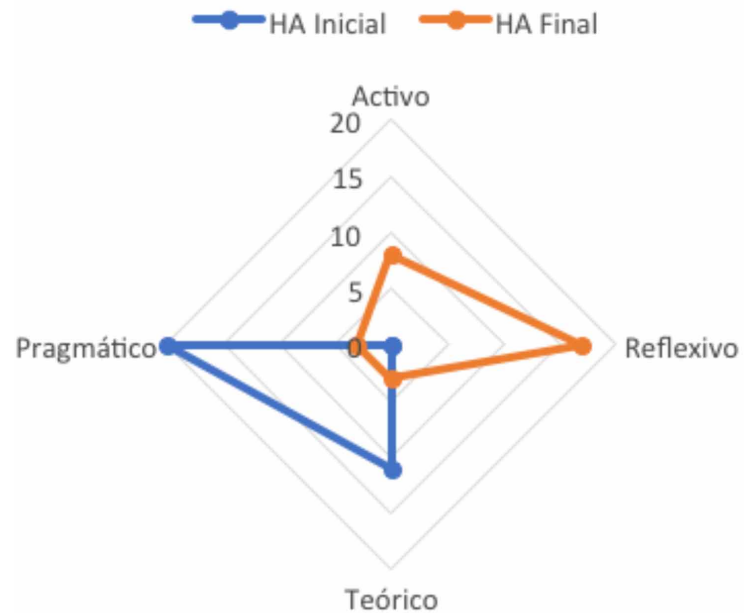

Fig. 1. Estilos de aprendizaje inicial y final en la gráfica de Honey-Alonso. Se observa la migración de Pragmáticos y Teóricos a Reflexivos y Activos. 
DREYER, E.; BARAHONA, P. \& CHÁVEZ, B. Estudiantes pragmáticos, teóricos y educación superior mediante metodologías activas. Int. J. Odontostomat., 15(1):152-158, 2021.

Tabla III. Aplicación del cuestionario Honey-Alonso inicial (HAI) y final (HAF) de los ABPs

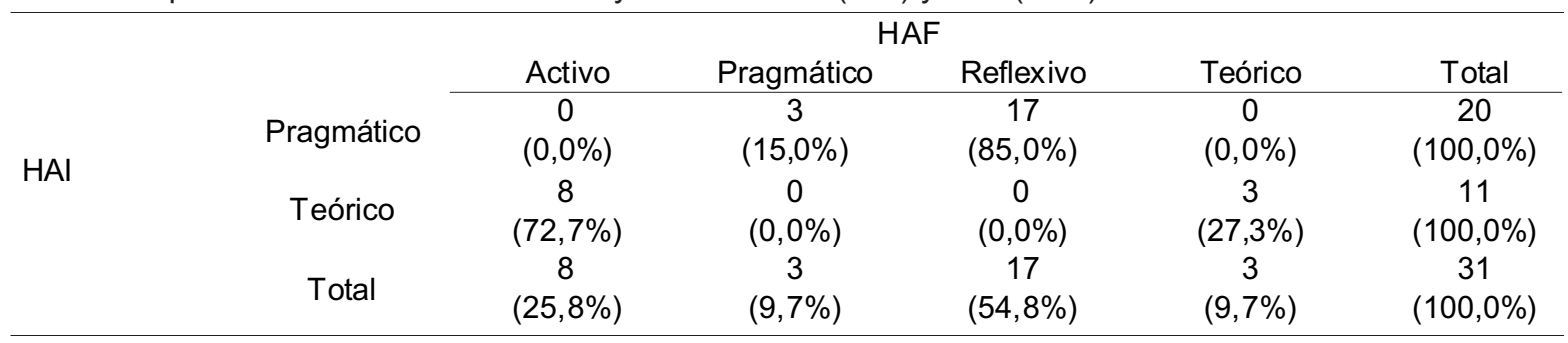

El tiempo directo asignado para la URPR fue de 50 horas, sin embargo, los ABPs requieren de tiempo indirecto para su desarrollo. Los tiempos totales invertidos en ABPs se pueden observar en la Tabla IV y su rango oscila desde 135 hasta198 horas. El tiempo indirecto autoreportado por los estudiantes y la evolución de estilos de aprendizaje, según género se puede observar en la Tabla V. En ella encontramos que el promedio de tiempo indirecto para las mujeres de la cohorte fue de 126 horas y para los hombres de 117 horas. Podemos observar diferencias por género en el uso de la metodología de los ABP, lo que significó gran cantidad de horas indirectas, cercano en promedio a 2,5 veces las horas directas. De los estudiantes pragmáticos iniciales $(n=20) 10$ hombres y 7 mujeres terminaron re-

Tabla IV. Tiempo total invertido por la cohorte en ABP expresado en horas

\begin{tabular}{|c|c|c|}
\hline \multicolumn{2}{|c|}{ Media } & 171,84 \\
\hline \multicolumn{2}{|c|}{ Desv. típ. } & $\begin{array}{c}18,223 \\
63\end{array}$ \\
\hline \multicolumn{2}{|c|}{ Mínimo } & 135 \\
\hline \multirow{4}{*}{ Percentiles } & 25 & 155 \\
\hline & 50 & 180 \\
\hline & 75 & 185 \\
\hline & & 198 \\
\hline
\end{tabular}

flexivos; 2 hombres y 1 mujer se mantuvieron en su perfil de pragmáticos.Por otro lado, de los estudiantes teóricos iniciales $(n=11), 3$ hombres y 5 mujeres terminaron como activos; 2 hombres y1 mujer se mantuvieron como teóricos.

En cuanto a la evolución de la cantidad de contenidos al inicio y término de los ABPs; de los contenidos disciplinares, sus medias y desviación típica para los contenidos disciplinares iniciales (DI) y disciplinares finales (DF) se pueden observar en la Tabla VI. En ella se observa que la media para contenidos disciplinares iniciales fue de 8,10 y finales de 13,71. La Figura 2, muestra la comparación de contenidos disciplinares iniciales vs finales por estudiante, en él podemos observar (con la excepción del estudiante número 23 , que mantuvo la misma cantidad de contenidos disciplinares) que la cohorte aumentó sus contenidos disciplinares al final de los ABPs. Su diferencia de medias fue estadísticamente significativa, $p=0,000$ (Tabla VII). Para los contenidos interdisciplinares, sus medias de contenidos Interdisciplinares Iniciales fue de 2,48 e interdisciplinares finales fue de 13,19 (Tabla VI). La Figura 3 compara los contenidos Interdisciplinares iniciales vs finales por estudiante, donde podemos visualizar que todos los estudiantes aumentaron noto-

Tabla V. Evolución de estilos de aprendizaje y promedios de tiempo indirecto utilizado, según género.

\begin{tabular}{lcccc}
\hline & \multicolumn{3}{c}{ Género } \\
\cline { 2 - 5 } Honey Alonso inicial/final & 10 & $59 \%$ & 7 & $50 \%$ \\
\hline Pragmático/Reflexivo & 2 & $12 \%$ & 1 & $7 \%$ \\
Pragmático/Pragmático & 2 & $12 \%$ & 1 & $7 \%$ \\
Teórico/Teórico & 3 & $17 \%$ & 5 & $36 \%$ \\
Teórico/Activo & $117 \mathrm{hrs}$. & $100 \%$ & $126 \mathrm{hrs}$. \\
Promedio tiempo indirecto en ABP & & & $100 \%$ \\
\hline
\end{tabular}

Tabla VI. Medias y desviación típica de contenidos disciplinares e interdisciplinares (iniciales y finales).

\begin{tabular}{ccccc}
\hline & \multicolumn{2}{c}{ Inicial } & \multicolumn{2}{c}{ Final } \\
\hline & Media & Desviación típ. & Media & Desviación típ. \\
\hline Disciplinares & 8,10 & 3,562 & 13,71 & 4,01 \\
Interdisciplinares & 2,48 & 1,151 & 13,19 & 4,9694 \\
\hline
\end{tabular}


DREYER, E.; BARAHONA, P. \& CHÁVEZ, B. Estudiantes pragmáticos, teóricos y educación superior mediante metodologías activas. Int. J. Odontostomat., $15(1): 152-158,2021$.

riamente sus contenidos interdisciplinares al final de su tarea. Su diferencia fue estadísticamente significativa, $p=0,000$ (Tabla VII ). La diferencia de medias de contenidos disciplinares F-I fue de 5,613, sin embargo, para los contenidos interdisciplinares F-I fue de 10,710, ambas diferencias fueron estadísticamente significativas (Tabla VIII). La Figura 4 muestra la comparación entre diferencias de contenidos disciplinares iniciales-finales vs interdisciplinares iniciales-finales. Se puede observar cómo los estudiantes al final de sus ABPs fueron capaces de desarrollar metodologías activas, independiente de su estilo de aprendizaje e incrementar significativamente más contenidos interdisciplinarios en contexto, para la toma de decisiones clínicas.
Tabla VII. Comparación de medias de contenidos Disciplinares e Interdisciplinares (iniciales respecto al final) mediante T-Test.

\begin{tabular}{lcccc}
\hline Contenidos & Comparación & $\mathrm{t}$ & $\begin{array}{c}\text { Sig. } \\
\text { (bilateral) }\end{array}$ & $\begin{array}{c}\text { Diferencia } \\
\mathrm{de}\end{array}$ \\
\hline Disciplinar & Inicial & 12,655 & $\mathrm{p}=0,000$ & 8,097 \\
& Final & 19,036 & $\mathrm{p}=0,000$ & 13,71 \\
Interdisciplinar & Inicial & 12,016 & $\mathrm{p}=0,000$ & 2,484 \\
& Final & 14,782 & $\mathrm{p}=0,000$ & 13,1935 \\
\hline
\end{tabular}

Tabla VIII. Comparación de diferencia de medias de contenidos disciplinares F-I vs interdisciplinares F-I mediante T-Test.

\begin{tabular}{lccc}
\hline Comparación & $\mathrm{t}$ & $\begin{array}{c}\text { Sig. } \\
\text { (bilateral) }\end{array}$ & $\begin{array}{c}\text { Diferencia } \\
\text { de medias }\end{array}$ \\
\hline $\begin{array}{l}\text { Diferencia Disciplinar } \\
\text { FI }\end{array}$ & 10,391 & $\mathrm{p}=0,000$ & 5,613 \\
$\begin{array}{l}\text { Diferencia } \\
\text { Interdisciplinar FI }\end{array}$ & 13,723 & $\mathrm{p}=0,000$ & 10,710 \\
\hline
\end{tabular}

\section{Contenidos disciplinares I/F}

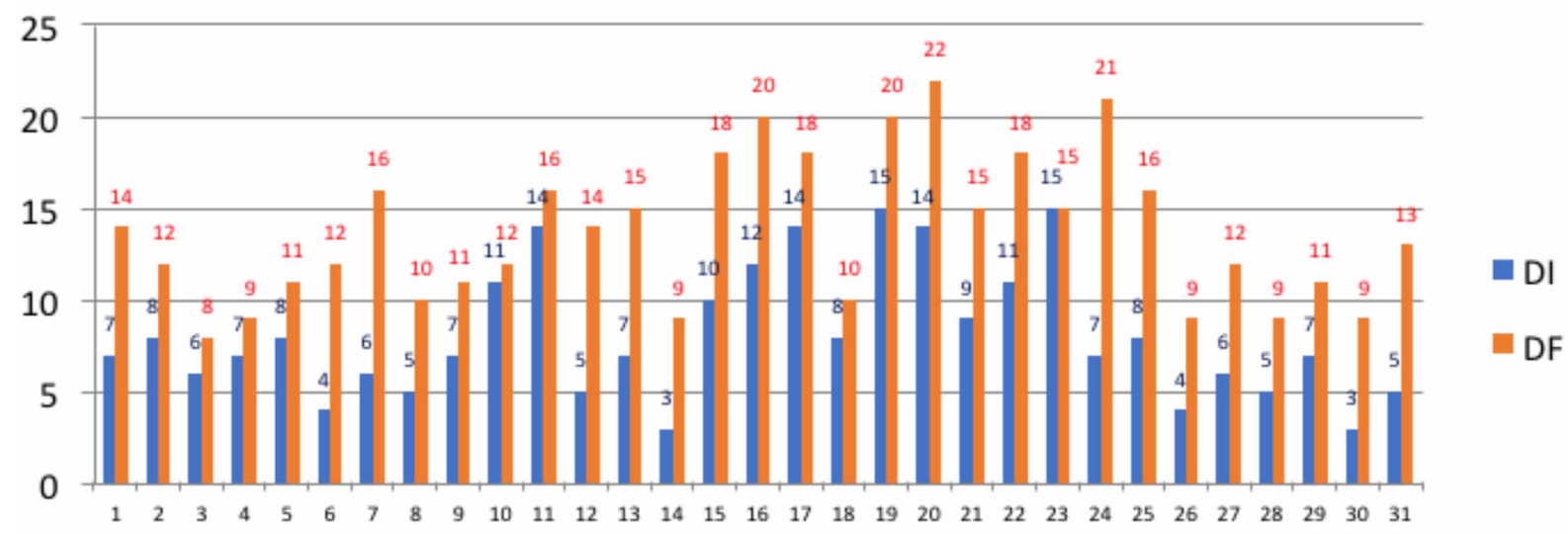

Estudiantes

Fig. 2. Comparación de contenidos disciplinares iniciales vs finales

Contenidos interdisciplinares I/F

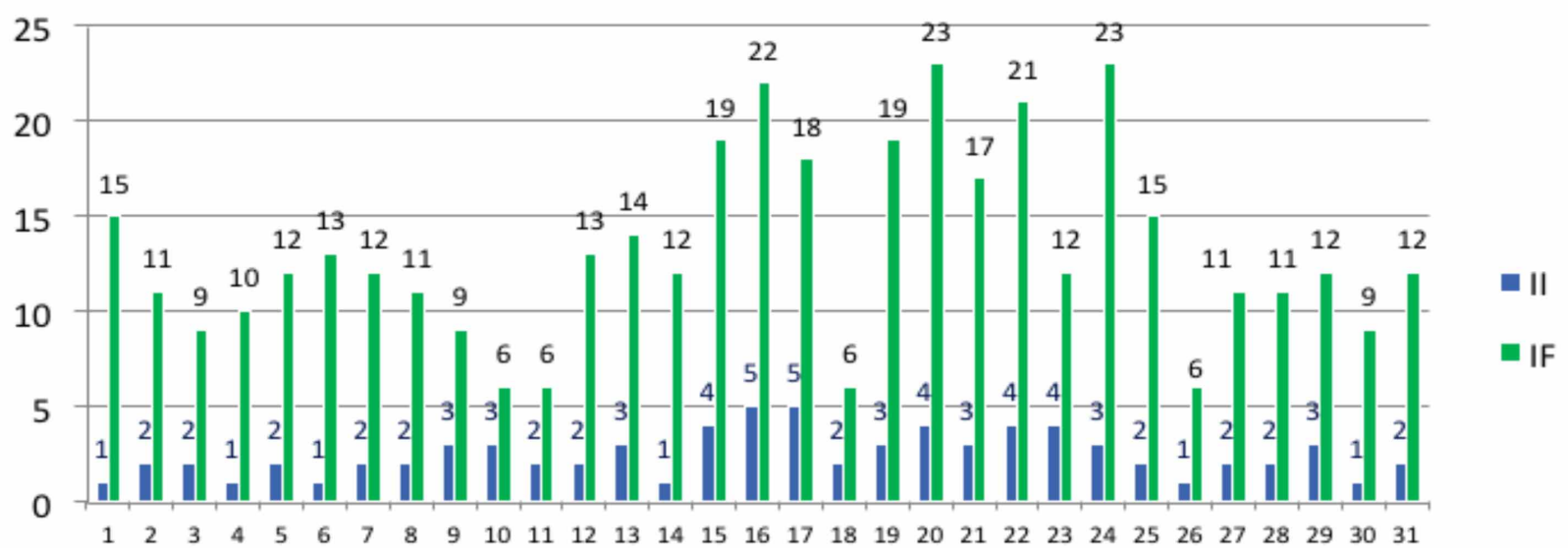

Estudiantes

Fig. 3. Comparación de contenidos Interdisciplinares iniciales vs finales. 


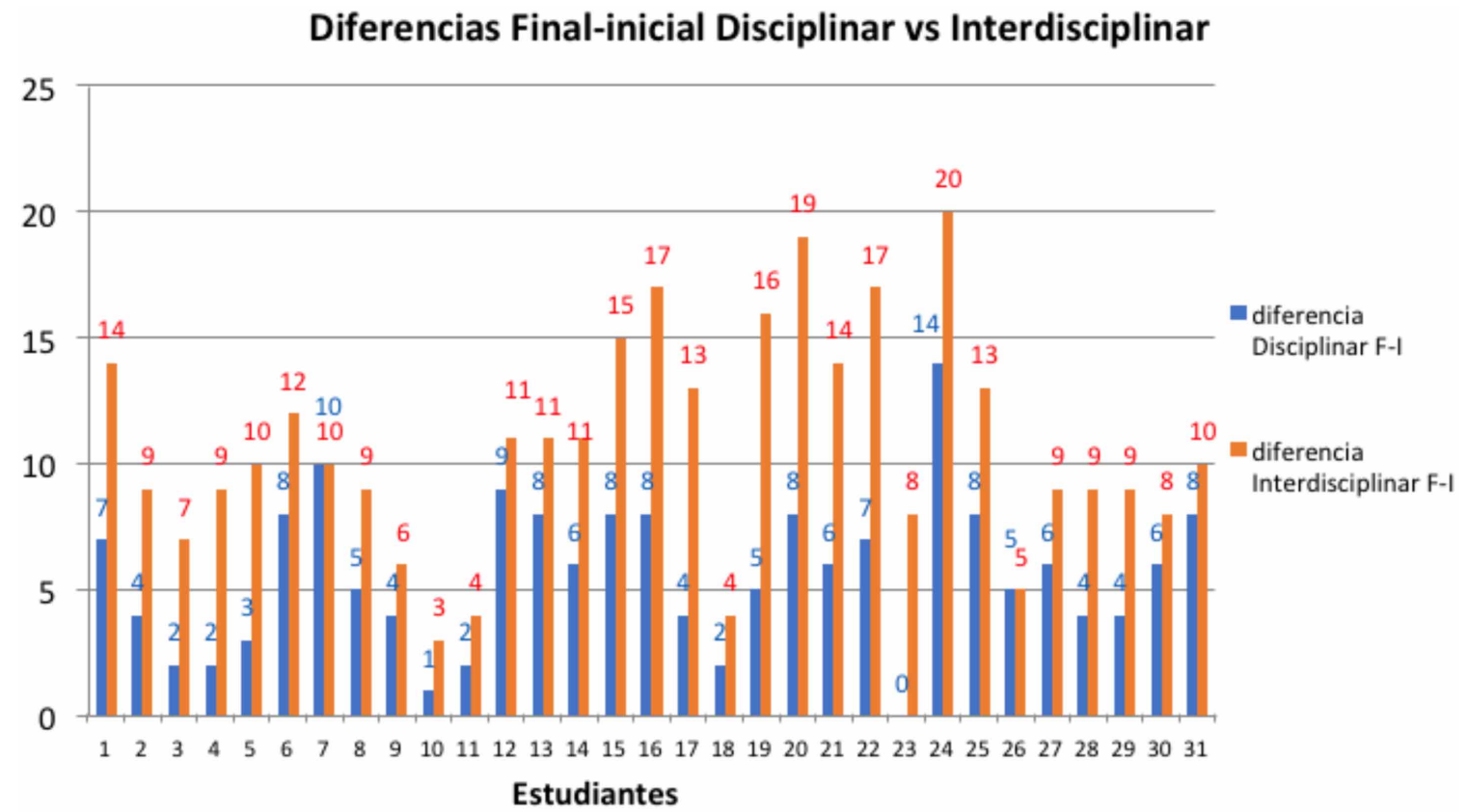

Fig. 4. Comparación entre Diferencias de contenidos disciplinares I-F vs interdisciplinares I-F

\section{DISCUSIÓN}

Existen numerosos problemas asociados a la comprensión de los estilos de aprendizaje. En primer lugar, hay una gran diferencia entre el modo en que alguien prefiere aprender y lo que realmente lleva a un aprendizaje eficaz y eficiente. En segundo lugar, la preferencia de cómo uno estudia, no es un estilo de aprendizaje. La mayoría de los llamados estilos de aprendizaje están basados en estereotipos, ellos, clasifican a las personas en grupos bien diferenciados, 30 según Coffield et al. (2004). La suposición que las personas se agrupan diferenciadamente, no obstante, tiene muy pocos estudios objetivos que lo sustenten. Finalmente, casi todos los estudios que presentan evidencia sobre estilos de aprendizaje, no satisfacen los criterios elementales de validez científica. Para algunos, los estilos de aprendizaje son solo un mito y la academia debería detener su difusión (Kirschner, 2017).

El fenómeno visualizado aquí, mediante el uso de $A R$ y $A B P$, es que la cohorte de estudiantes que voluntariamente decidió participar de este ensayo, profesionales Cirujano-Dentistas, al comprender el problema clínico de manera asistida por el docente, respetando sus tiempos de desarrollo, evolución y productividad, descubrieronla pertinencia y coherencia de su tarea, independiente de su denominado estilo de aprendizaje. Estudiantes teóricos y pragmáticos por definición, no podrían, por la naturaleza de su estilo de aprendizaje, estar dispuestos a desarrollar metodologías activas y, sin embargo, todos ellos las desarrollaron, incluso quienes permanecieron en su clasificación inicial de estilo de aprendizaje, una vez terminada la URPR.Los estudiantes identificaron sus necesidades de aprender, dependiente del contexto y aplicaron sus nuevos conocimientos para la solución de los problemas. Este proceso reflejó lo aprendido y validó la efectividad de la estrategia utilizada. Las metodologías activas como el $A R$ y $A B P$, requieren tiempo directo e indirecto. Este reporte informa por primera vez el tiempo requerido por una cohorte de profesionales para lograr su tarea. El tiempo indirecto,a lo menos duplica las horas directas asignadas. Este dato es relevante al momento de construir programas y certificar horas formativas.

Por otra parte, los estudiantes comprendieron la totalidad de la problemática de sus casos, incorporando contenidos más allá de la disciplina que estudiaron. Esto se visualizó con un incremento significativo del uso de contenidos interdisciplinares al final de sus ABPs, como respaldo de las decisiones terapéuticas tomadas. Con ello, la cohorte fue capaz de proponer y ejecutar, de manera autónoma, soluciones basadas en evidencia, que resuelven la totalidad de las necesidades de las enfermedades y condiciones requeridas por sus pacientes, en el marco del programa de especialización en periodoncia de la Universidad de Chile. 
DREYER, E.; BARAHONA, P. \& CHÁVEZ, B. Pragmatists, theorists' students and tertiary education through active methodologies. Int. J. Odontostomat. 15(1):152-158, 2021.

ABSTRACT: New conceptual models for health education emphasize content and context relevance for significant therapeutic interventions. Students should actively be involved in learning. One way to promote this are the active learning methodologies. Students educated by Reverse Classroom (RC) and Problem Based Learning (PBL) exhibit superior professional skills and effective learning. According to Honey-Alonso(H-A), there are four learning styles: Theorists $(T)$ Pragmatists $(P)$ Reflective (R) and Actives (A). The objective was to apply active methodologies in professionals, knowing their learning style, while they are students in a specialty program. The Periodontics program at the Dental School, Universidad de Chile, has a rehabilitation unit (RU). Between 2009 and 2019 the programme had 67 students, 31 agreed to participate signing the consent form. RC and PBL were applied in RU for the following contents: Caries Risk assessment, ferrule, muscle deprogramming, occlusal adjustment, teeth retention and implant site selection. First and last PBL presentations were compared categorizing disciplinary and interdisciplinary contents in contexts. At the same time, the HA questionnaire was applied to all participants. Collected data was analyzed in SPSS v.21 program. For the variables disciplinary, interdisciplinary contents, t test was applied with significance $\leq 0.05$. The cohort $n=31 ; 14$ women (mean 30 years), 17 men (mean29 years). Basel $H A=20(P)$ and 11(T). Final $\mathrm{H} \mathrm{A}=8(\mathrm{~A}), 3(\mathrm{P}), 17(\mathrm{R}), 3(\mathrm{~T})$. Median for disciplinary contents at basal was 8.1 and final was $13.71, p=0.00$. Median for interdisciplinary contents at basal was 2.48 and at final was 13.19, $p=0.00$. Median differences for disciplinary contents finalbasal $=5.63$, for interdisciplinary contents final-basal= 10.71 , $p=0.00$. All students engage actives methodologies, regardless of their learning style significantly increased more interdisciplinary contents in context for clinical decision making.

KEY WORDS: problem-based learning, dental education, content, context.

\section{REFERENCIAS BIBLIOGRÁFICAS}

Alrahlah, A. How effective the problem-based learning (PBL) in dental education. A critical review. Saudi Dent. J., 28(4):155-61, 2016.

Auclair, F. Problem formulation by medical students: an observation study. BMC Med. Educ., 7:16, 2007.

Balogh, E. P.; Miller, B. T. \& Ball, J. R. Improving Diagnosis in Health Care. National Academies of Sciences, Engineering, and Medicine. Washington (DC), The National Academies Press, 2015.

Cindy E. Hmelo-Silver. Problem-based learning: what and how do students learn? Educ. Psychol. Rev., 16:235-66, 2004.

Christensen, N.; Black, L.; Furze, J.; Huhn, K.; Vendrely, A. \& Wainwright, S. Clinical reasoning: survey of teaching methods, integration, and assessment in entry-level physical therapist academic education. Phys. Ther., 97(2):175-86, 2017.

Coffield, F.; Moseley, D., Hall, E. \& Ecclestone, K. Learning Styles and Pedagogy in Post-16 Learning: A Systematic and Critical Review. Londres, Learning and Skills Research Centre, 2004.

Cutrer, W. B.; Miller, B.; Pusic, M. V.; Mejicano, G.; Mangrulkar, R. S.;
Gruppen, L. D.; Hawkins, R. E.; Skochelak, S. E. \& Moore Jr., D. E. Fostering the development of master adaptive learners: a conceptual model to guide skill acquisition in medical education. Acad. Med., 92(1):70-5, 2017.

Dalton, L.; Gee, T. \& Levett-Jones, T. Using clinical reasoning and simulation-based education to 'flip' the Enrolled Nurse curriculum. Aust. J. Adv. Nurs., 33(2):28-34, 2015.

de Bruin, A. B. H.; Schmidt, H. G. \& Rikers, R. M. J. P. The role of basic science knowledge and clinical knowledge in diagnostic reasoning: a structural equation modeling approach. Acad. Med., 80(8):76573,2005

Forés, A.; Gamo, J. R.; Guillén, J. C.; Hernández, T.; Ligioiz, M.; Pardo, F. \& Trinidad C. Neuromitos en Educación. Barcelona, Plataforma, 2015.

Glick, M.; Williams, D. M.; Kleinman, D. V.; Vujicic, M.; Watt, R. G. \& Weyant, R. J. Reprint of: A new definition for oral health supported by FDI opens the door to a universal definition of oral health. $J$. Dent., 57:1-3, 2017.

Gruppen, L. D. Clinical reasoning: defining it, teaching it, assessing it, studying it. West. J. Emerg. Med., 18(1):4-7, 2017.

Khin-Htun, S. \& Kushairi, A. Twelve tips for developing clinical reasoning skills in the pre-clinical and clinical stages of medical school. Med. Teach., 41(9):1007-11, 2019.

Kirschner, P. A. Stop propagating the learning styles myth. Comput. Educ., 106:166-71, 2017.

Martínez, M. Un Camino Innovador Construido por los Docentes de las Universidades: Una Visión Analítica. En: Carrasco, C. \& Corral, I. (Eds.). Docencia Universitaria e Innovación. Evolución y Retos a través de los CIDIU. Barcelona, Octaedro, 2018. pp.53-71.

Naranjo, C. Conocimiento Didáctico del Contenido en Profesores de Odontología bien Considerados por la Comunidad Académica. Barcelona, Universitat de Barcelona, 2018.

Novak, K.; Mandin, H.; Wilcox, E. \& McLaughlin, K. Using a conceptual framework during learning attenuates the loss of expert-type knowledge structure. BMC Med. Educ., 6:37, 2006.

The Flipped Classroom. Experiencias y Recursos para Dar "La Vuelta" a la Clase. Website. The Flipped Classroom, 2019. Disponible en: www.theflippedclassroom.es

Tovar-Moncada, M. C. \& Crespo-Knopfler, S. Del aprendizaje vicario al aprendizaje reflexivo en la formación profesional de enfermería. Rev. Enf. Inst. Mex. Seguro Soc., 23(2):115-20, 2015.

Varpio, L.; O'Brien, B.; Hu, W.; Ten Cate, O.; Durning, S. J.; van der Vleuten, C.; Gruppen, L.; Irby, D.; Humphrey-Murto, S. \& Hamstra, S. J. Exploring the institutional logics of health professions education scholarship units. Med. Educ., 51(7):755-67, 2017.

Woods, N. N.; Brooks, L. R. \& Norman, G. R. It all make sense: biomedical knowledge, causal connections and memory in the novice diagnostician. Adv. Health Sci. Educ. Theory Pract., 12(4):405-15, 2007.

Yazdani, S.; Hosseinzadeh, M. \& Hosseini, F. Models of clinical reasoning with a focus on general practice: A critical review. J. Adv. Med. Educ. Prof., 5(4):177-84, 2017

Dirección de correspondencia

Erik Dreyer Arroyo

Cirujano-Dentista, PhD

Especialista en Rehabilitación Oral.

Departamento de Odontología Conservadora

Facultad de Odontología

Universidad de Chile.

Santiago - CHILE

E-mail: edreyer@odontologia.uchile.cl 